\title{
ON BOCHNER-KRALL ORTHOGONAL POLYNOMIAL SYSTEMS
}

\author{
T. BERGKVIST, H. RULLGÅRD and B. SHAPIRO
}

\begin{abstract}
In this paper we address the classical question going back to S. Bochner and H. L. Krall to describe all systems $\left\{p_{n}(x)\right\}_{n=0}^{\infty}$ of orthogonal polynomials (OPS) which are the eigenfunctions of some finite order differential operator. Such systems of orthogonal polynomials are called Bochner-Krall OPS (or BKS for short) and their spectral differential operators are accordingly called Bochner-Krall operators (or BK-operators for short). We show that the leading coefficient of a Nevai type BK-operator is of the form $((x-a)(x-b))^{N / 2}$. This settles the special case of the general conjecture 7.3 of [4] describing the leading terms of all BK-operators.
\end{abstract}

\section{Summary}

Consider a sequence of polynomials $\left\{p_{n}\right\}_{n=0}^{\infty}$ in a variable $x$, where deg $p_{n}=n$. This sequence is orthogonal with respect to a measure $\mu$ if $\int p_{n}(x) p_{m}(x) d \mu(x)$ is nonzero precisely when $n=m$. We are here concerned with polynomials orthogonal with respect to a measure of the so-called Nevai class, see [11] and below. Furthermore we say that $\left\{p_{n}\right\}$ is a sequence of eigenpolynomials if there exists a differential operator $\mathfrak{D}=\sum_{k=1}^{N} a_{k}(x)(d / d x)^{k}$ where $a_{k}$ are polynomials in $x$. Finally, $\left\{p_{n}\right\}$ is called a Bochner-Krall system if it is both orthogonal and a system of eigenpolynomials. If it is orthogonal with respect to a measure of Nevai class, we say that it is a Bochner-Krall system of Nevai type.

It is an open problem to classify all Bochner-Krall systems. In [4] it is conjectured that the leading coefficient $a_{N}$ for any Bochner-Krall system is a power of a polynomial of degree at most 2 . Our main result is an affirmative answer to this conjecture for Bochner-Krall systems of Nevai type.

MAIN THEOREM. Let $\left\{p_{n}\right\}$ be a compact type BKS, orthogonal with respect to a measure $\mu$ and with differential operator $\mathrm{D}$. If $\mu$ is of the Nevai class and the convex hull of $\operatorname{supp} \mu$ is the interval $[a, b]$, then $N$ is even and $a_{N}(x)$ is a constant multiple of $((x-a)(x-b))^{N / 2}$. 
This result is obtained by combining the results on the asymptotic distribution of zeros from [1] and [11].

\section{Introduction}

Let $P_{\mathrm{R}}$ and $P_{\mathrm{C}}$ denote the spaces of all real and complex polynomials, respectively, in a variable $x$. By a real (or complex) polynomial system we will mean a sequence $\left\{p_{n}\right\}_{n=0}^{\infty}$ of polynomials in $P_{\mathrm{R}}$ (or $P_{\mathrm{C}}$ ) such that deg $p_{n}=n$. By an orthogonal polynomial system (OPS) one understands a real polynomial system $\left\{p_{n}\right\}$ such that $\left\langle p_{n}, p_{m}\right\rangle$ is nonzero precisely when $n=m$, where $\langle$,$\rangle is some$ reasonable inner product on the linear space $P_{\mathrm{R}}$. If an orthogonal polynomial system for a given inner product exists, the $p_{n}$ are unique, up to multiplication by scalars.

Orthogonal polynomial systems have been studied in various degrees of generality. Classically, one has considered inner products of the form

$$
\langle p, q\rangle=\sigma(p \cdot q)
$$

where $\sigma$ is a moment functional, that is a linear functional on $P_{\mathrm{R}}$. It is known that all moment functionals can be represented by an integral

$$
\sigma(p)=\int p(x) d \mu(x)
$$

where $\mu$ is a (possibly signed) Borel measure on the real line. The most complete theories have been obtained in the case where $\mu$ is positive with compact support, and moreover belongs to the so-called Nevai class. If the density of $\mu$ is a function $\rho$ and $\log \rho$ is integrable on the smallest interval containing $\operatorname{supp} \mu$, then $\mu$ is of the Nevai class, but this condition is not necessary. See [11] for the precise definition of the Nevai class, denoted there by $M(a, b)$. In what follows we will mainly be concerned with orthogonal polynomial systems of this particular kind, which we will call orthogonal systems of Nevai type. Recently, there has been interest in more general inner products called Sobolev, which are of the form

$$
\langle p, q\rangle=\sum_{k=0}^{M} \sigma_{k}\left(p^{(k)} \cdot q^{(k)}\right)
$$

where the $\sigma_{k}$ are moment functionals. For the basics of the classical theory of orthogonal polynomials see e.g. [12] and [3].

Consider now a differential operator

$$
\mathfrak{D}=\sum_{k=1}^{N} a_{k}(x) \frac{d^{k}}{d x^{k}}
$$


where the coefficients $a_{k}(x)$ are polynomials in $P_{\mathrm{C}}$. We are interested in eigenpolynomials of this operator, that is polynomials $p \in P_{\mathrm{C}}$ satisfying $\delta p=\lambda p$ for some constant $\lambda$. Already $S$. Bochner observed that the operator $D$ has infinitely many linearly independent eigenpolynomials if and only if $\operatorname{deg} a_{k}(x) \leq k$, with equality for at least one $k$. In this case there is precisely one monic degree $n$ eigenpolynomial $p_{n}$ for all sufficiently large $n$. For generic $a_{k}(x)$, the same is true for every $n \geq 0$. If a complex polynomial system consists of eigenpolynomials for an operator of the form (2), we will call it a system of eigenpolynomials.

A Bochner-Krall system (BKS for short) is defined to be a real polynomial system which is both orthogonal (with respect to some inner product $\langle$,$\rangle ) and$ a system of eigenpolynomials (for some differential operator $\mathfrak{D}$ ). In this case $\langle$,$\rangle is called a Bochner-Krall inner product, and \mathfrak{D}$ is called a Bochner-Krall operator. If a BKS is an orthogonal system of Nevai type, we will call it for short a Nevai type BKS. The results we report in this note are valid for all Nevai type BKS.

It is an open problem to classify all Bochner-Krall systems. A complete classification is only known for Bochner-Krall operators $D$ with $N \leq 4$. The corresponding BKS are various classical systems such as the Jacobi-type, the Laguerre-type, the Legendre-type, the Bessel and the Hermite polynomials, see [4], Th. 3.1. In general, it is not even known which differential operators are Bochner-Krall operators for some BKS. In [4] it is conjectured that the leading coefficient $a_{N}(x)$ of a Bochner-Krall operator is a power of a polynomial of degree at most 2. Our main result is an affirmative answer to this conjecture for Nevai type BKS.

Our results are obtained by studying the asymptotic distribution of zeros of a polynomial system. To make this more precise, let $\left\{p_{n}\right\}$ be a polynomial system and for fixed $n \geq 1$, let $\alpha_{1}, \ldots, \alpha_{n}$ denote the (real or complex) zeros of $p_{n}$. Let $v_{n}=\frac{1}{n} \sum_{i=1}^{n} \delta\left(x-\alpha_{i}\right)$ be the probability measure in the complex plane with point masses at these zeros. We call the measures $v_{n}$ the root measures of the polynomial system $\left\{p_{n}\right\}$. If the sequence of root measures $v_{n}$ converges weakly to a measure $v$ when $n \rightarrow \infty$, we say that $v$ is the asymptotic distribution of zeros of the polynomial system.

The following results, which characterize the asymptotic distribution of zeros for Nevai type OPS and for systems of eigenpolynomials respectively, are crucial to our treatment.

Suppose that a polynomial system $\left\{p_{n}\right\}$ is orthogonal with respect to a positive measure $\mu$, and that the convex hull of $\operatorname{supp} \mu$ is a compact interval $[a, b]$. It is well known (see [3]) that the zeros of every $p_{n}$ are contained in the interval $[a, b]$. The following is a more precise result on the distribution of zeros for orthogonal systems of Nevai type. 
Theorem A (see [11], Th. 3, p. 50). Let the polynomial system $\left\{p_{n}\right\}$ be orthogonal with respect to a measure $\mu$ of Nevai class on $\mathrm{R}$, and let the convex hull of $\operatorname{supp} \mu$ be $[a, b]$. Then the asymptotic distribution of zeros of $\left\{p_{n}\right\}$ is an absolutely continuous measure $v$ which depends only on $[a, b]$. The support of $v$ is precisely $[a, b]$ and its density in this interval is given by

$$
\rho(x)=\frac{1}{\pi \sqrt{(b-x)(x-a)}} .
$$

Next we describe the asymptotic distribution of zeros for a system of eigenpolynomials.

THeOREM B (see [1] Th. 2 and 4). Let $\left\{p_{n}\right\}$ be a system of eigenpolynomials for an operator o with $a_{N}(x)$ monic of degree $N$. Then the asymptotic distribution of zeros of $\left\{p_{n}\right\}$ is a probability measure $v$ with the following properties:

a) $v$ has compact support;

b) its Cauchy transform $C(x)=\int \frac{d v(\zeta)}{x-\zeta}$ satisfies the equation $C(x)^{N}=$ $1 / a_{N}(x)$ for almost all $x \in \mathrm{C}$.

These properties determine $v$ uniquely.

Note that the limiting measure $v$ is independent of all terms in (2) except the leading term $a_{N}(x) \frac{d^{N}}{d x^{N}}$.

To derive from these two results a statement about Nevai type BKS, we will need the following.

Proposition 1. Let $\left\{p_{n}\right\}$ be a system of eigenpolynomials for a differential operator $\mathfrak{D}$, and assume that all the zeros of $p_{n}$ are real. Then there exists a compact set containing all the zeros of every $p_{n}$ if and only if $\operatorname{deg} a_{N}(x)=N$.

Now it is easy to derive the following

MAIN Theorem. Let $\left\{p_{n}\right\}$ be a Nevai type BKS, orthogonal with respect to a measure $\mu$ and with differential operator $\mathrm{D}$. If $\mu$ is of the Nevai class and the convex hull of $\operatorname{supp} \mu$ is the interval $[a, b]$, then $N$ is even and $a_{N}(x)$ is a constant multiple of $((x-a)(x-b))^{N / 2}$.

REFERENCES AND ACKNOWLEDGEMENTS. There exists a really vast literature devoted to the classification problem for OPS. Classification of BKS has also attracted considerable attention, see e.g. [4] with its 100 references and [7], [8], [9] and references therein. We are grateful to Dr. M. Shapiro and Professor H. Shapiro for a number of discussions on the topic. We shold point out that when a preliminary version of this note was shown to some of the experts in 
this field, we were sent a paper in preparation [6] containing results very much in the same spirit as this note.

\section{Proofs}

We need to prove Proposition 1 and the main theorem (as its corollary). Since the proof of Theorem B, in the situation where we will need it, follows easily along the same lines, we will include such a proof for the convenience of the reader.

Consider a polynomial system $\left\{p_{n}\right\}$ with the associated root measures $v_{n}$. Assume that the supports of the measures $v_{n}$ are all contained in the same compact set, and that $v_{n} \rightarrow v$ in the weak topology. Let $C_{n}(x)$ be the Cauchy transform of $v_{n}$ and note that

$$
C_{n}(x)=\int \frac{d v_{n}(\zeta)}{x-\zeta}=\frac{p_{n}^{\prime}(x)}{n p_{n}(x)}
$$

If $C(x)$ denotes the Cauchy transform of $v$, it follows that

$$
\frac{p_{n}^{\prime}(x)}{n p_{n}(x)} \rightarrow C(x)
$$

for almost every $x \in \mathrm{C}$.

Suppose now that $\left\{p_{n}\right\}$ is a system of eigenpolynomials for an operator $\grave{D}$ and that $\operatorname{supp} v_{n}$ are all contained in the same compact subset of the real line. Then there exists at least a subsequence of the $v_{n}$ converging weakly to some measure $v$. Moreover, if we let $v_{n}^{(j)}$ denote the root measure of the $j$ th derivative of $p_{n}$, then it follows from Rolle's theorem that (a subsequence of) $v_{n}^{(j)}$ converges weakly to $v$ for every $j>0$. In particular,

$$
\frac{p_{n}^{(j+1)}(x)}{(n-j) p_{n}^{(j)}(x)} \rightarrow C(x)
$$

for almost every $x \in \mathrm{C}$, where $C(x)$ is the Cauchy transform of $v$. If we divide both sides of the differential equation $\mathrm{D} p_{n}=\lambda_{n} p_{n}$ by $n(n-1) \ldots(n-N+1) p_{n}$ we obtain

$$
\begin{array}{r}
a_{N}(x) \prod_{j=0}^{N-1} \frac{p_{n}^{(j+1)}(x)}{(n-j) p_{n}^{(j)}(x)}+\frac{a_{N-1}(x)}{n-N+1} \prod_{j=0}^{N-2} \frac{p_{n}^{(j+1)}(x)}{(n-j) p_{n}^{(j)}(x)}+\cdots \\
=\frac{\lambda_{n}}{n(n-1) \ldots(n-N+1)} .
\end{array}
$$


When $n \rightarrow \infty$ all the terms on the left hand side but the first one tend to zero, and so

$$
a_{N}(x) C(x)^{N}=\lim _{n \rightarrow \infty} \frac{\lambda_{n}}{n(n-1) \ldots(n-N+1)}
$$

for almost all $x$. But it can be seen (see [1]) that $\lambda_{n}=\sum_{k=1}^{N} c_{k} n(n-1) \ldots(n-$ $k+1)$ where $c_{k}$ is the coefficient at $x^{k}$ in $a_{k}(x)$. In particular, if deg $a_{N}(x)<N$ i.e. $c_{N}=0$, then $\lambda_{n} / n(n-1) \ldots(n-N+1) \rightarrow 0$ when $n \rightarrow \infty$, and it follows that $C(x)=0$ for almost all $x$. This implies that $v=0$, a contradiction. This argument proves one of the implications in Proposition 1. For the other implication we refer to [1], Lemma 9. Moreover, if $a_{N}(x)$ is monic of degree $N$, then $\lambda_{n} / n(n-1) \ldots(n-N+1) \rightarrow 1$, and it follows that $C(x)^{N}=1 / a_{N}(x)$.

Suppose now that $\left\{p_{n}\right\}$ is a Nevai type BKS as in the main theorem. By the remark preceeding Theorem $\mathrm{A}$, the zeros of every $p_{n}(x)$ are contained in the interval $[a, b]$. It follows from Proposition 1 that $\operatorname{deg} a_{N}(x)=N$, and we might as well assume that $a_{N}(x)$ is monic. Hence the Cauchy transform $C(x)$ of the limit $v=\lim _{n \rightarrow \infty} v_{n}$ satisfies $C(x)^{N}=1 / a_{N}(x)$. On the other hand, a direct computation of the Cauchy transform, using the expression for $v$ in Theorem A, gives $C(x)^{2}=1 /(x-a)(x-b)$. Comparing these results yields $a_{N}(x)=((x-a)(x-b))^{N / 2}$.

\section{Final remarks}

PRoBlem 1. The major problem in the context of this paper is whether every BKS, orthogonal with respect to a positive mesure with compact support, is a Jacobi-type OPS, compare [4], Conjecture 7.3. For the constant leading coefficient the analogous fact was proved in [9].

Problem 2. Is there an analog of Theorem A on the asymptotic zero distribution for a signed measure $\mu$ with compact support? What is the situation for a probability measure with a noncompact support as well as for Sobolev orthogonal polynomial systems. (There exists a literature on this topic.)

Problem 3. Generalize the results of [1] to operators with $\operatorname{deg} a_{N}(x)<N$. Preliminary computer experiments show that similar results on the asymptotic distribution of zeros would hold for all operators (2).

\section{REFERENCES}

1. Bergkvist, T., and Rullgård, H., On polynomial eigenfunctions for a class of differential operators, Math. Res. Lett 9 (2002), 153-171.

2. Bochner, S., Über Sturm-Liouvillesche Polynomsysteme, Math. Z. 89 (1929), 730-736.

3. Chihara, T., An Introduction to Orthogonal Polynomials, Ser. Math. \& Appl. 13, Gordon \& Breach, 1978. 
4. Everitt, W. N., Kwon, K. H., Littlejohn, L. L., and Wellman, R., Orthogonal polynomial solutions of linear ordinary differential equations, J. Comput. Appl. Math 133 (2001), 85-109.

5. Krall, H. L., On orthogonal polynomials satisfying a certain fourth order differential equation, The Pennsylvania State College Studies, No 6, 1940.

6. Kwon, K. H., and Lee, D. W., Characterization of Bochner-Krall orthogonal polynomials of Jacobi type, preprint.

7. Kwon, K. H., Littlejohn, L. L., and Yoon, G. J., Bochner-Krall orthogonal polynomials, Special functions (Hong-Kong, 1999), 181-193, World Sci. Publ., River Edge, NJ, 2000.

8. Kwon, K. H., Littlejohn, L. L., and Yoon, G. J., Orthogonal polynomial solutions to spectral type differential equations: Magnus's conjecture, J. Approx. Theory 112 (2001) 2, 189215.

9. Kwon, K. H., Yoo, B. H., and Yoon, G. J., A characterization of Hermite polynomials, J. Comput. Appl. Math. 78 (1997), 295-299.

10. Másson, G., and Shapiro, B., A note on polynomial eigenfunctions of a hypergeometric type operator, Experiment. Math. 10 (2001), 4, 609-618.

11. Nevai, P., Orthogonal polynomials, Mem. Amer. Math. Soc. 213 (1979), 1-185.

12. Szegö, G., Orthogonal Polynomials, Amer. Math. Soc. Colloq. Publ. 23, 1978.

DEPARTMENT OF MATHEMATICS

UNIVERSITY OF STOCKHOLM

S-10691 SWEDEN

E-mail: tanjab@mat.su.se, hansr@mat.su.se, shapiro@mat.su.se 